\title{
Obst- und Gartenbau unter Kurfürst August von Sachsen (1526-1586)
}

\author{
Christine Nagel
}

$\mathrm{D}$ er Übergang der Kurfürstenwürde von den Ernestinern an die Albertiner im Jahr 1547 war auch für den fürstlichen Obst- und Gartenbau in Sachsen von Bedeutung. Denn mit diesem Ereignis gelangten zwei ernestinische Residenzen, Schloss Hartenfels in Torgau und das Jagdschloss Lochau, in den Besitz der Albertiner. Beide Wohnsitze verfügten über Gartenanlagen, die unter den ernestinischen Herrschern angelegt worden waren. So hatte Friedrich der Weise in Lochau einen ummauerten Garten mit Weinanbau, Obstbäumen, Kräutern, kleinen Lusthäusern und Teichen anlegen lassen. ${ }^{1}$ Johann Friedrich der Großmütige, der Schloss Hartenfels in Torgau ab 1533 zu einem der schönsten deutschen Renaissanceschlösser umbaute, umgab dieses ebenfalls mit fürstlichen Gartenanlagen. ${ }^{2}$

Der albertinische Kurfürst Moritz wählte sich jedoch Dresden zur Hauptresidenz und ließ in der Nähe des Residenzschlosses »Lustgärten $\aleph^{3}$ anlegen. Anregungen und junge Obstbäume erbat er sich hierzu vom kaiserlichen Hofgärtner in Prag. ${ }^{4}$ Als Kurfürst Moritz 1553 starb, verfügte sein Bruder und Nachfolger August über Gärten in Dresden, Torgau und Lochau. Er stellte hierfür ausländische Spezialisten an. Ein niederländischer Gärtner versorgte die Anlagen in Torgau und Lochau. Für Dresden wurde ein französischer Gärtner eingestellt. ${ }^{5}$

Kurfürst August und dessen Gemahlin Anna interessierten sich zunächst ganz allgemein für die Gartenkunst. Sie erbaten sich von befreundeten Fürsten und auch vom Kaiser vor allem "gute fremde Samen von Kräutern, Früchten, Blumen«. ${ }^{6}$ Diese
Wünsche nach »fremden Samen « sind in der Korrespondenz meist nicht spezifiziert. Alles, was irgendwie ungewöhnlich und exotisch erschien, war erwünscht. Ein Zeichen dieses Interesses ist auch das 1563 im Auftrag des Kurfürsten von Johannes Kentmann zusammengetragene »Kreutterbuch «.7 Die über 600 Einträge sind mit Zeichnungen des Malers David Redtel illustriert. Neben einheimischen Pflanzen, Sträuchern und Bäumen sind darin auch Gewächse abgebildet, die üblicherweise nicht im sächsischen Raum vorkommen. Hierzu zählen die Tomaten- und die Paprikapflanze, die Lilie, der Granatapfel- und der Zitronenbaum (Abb. 1). Einige der exotischen Pflanzen fand Johannes Kentmann, der als Stadtphysikus in Torgau angestellt war, in den beiden bedeutenden botanischen Gärten in Torgau und Meißen. ${ }^{8}$

Auf Dauer war das Kurfürstenpaar mit seinen ausländischen Gärtnern nicht zufrieden. Dies legt ein Schreiben der Kurfürstin Anna an den Nürnberger Kaufmann Martin Pfinzing vom 3. September 1568 nahe. Sie schrieb, dass sie Bedenken habe, "einigen franzosen und niederländern mehr ihre gärten anzuvertrauen, sondern bedacht sei, dieselben hinfüro mit deutschen gärtnern und arbeitern bestellen zu lassen «. ${ }^{9}$ Sie bat den Kaufmann, ihr bei der Suche behilflich zu sein. Mit der Bestallung von Georg Winger aus Nürnberg, der bis dahin in Diensten der Landgräfin Mechthild von Leuchtenburg gestanden hatte, am 30. September 1568 hatte das Kurfürstenpaar einen tüchtigen Hofgärtner gewonnen. ${ }^{10}$ Er war für die Anlagen in Dresden inklusive des Vorwerks Ostra, das als Musterwirtschaft zur Versorgung 
Obst- und Gartenbau unter Kurfürst August von Sachsen (1526-1586)

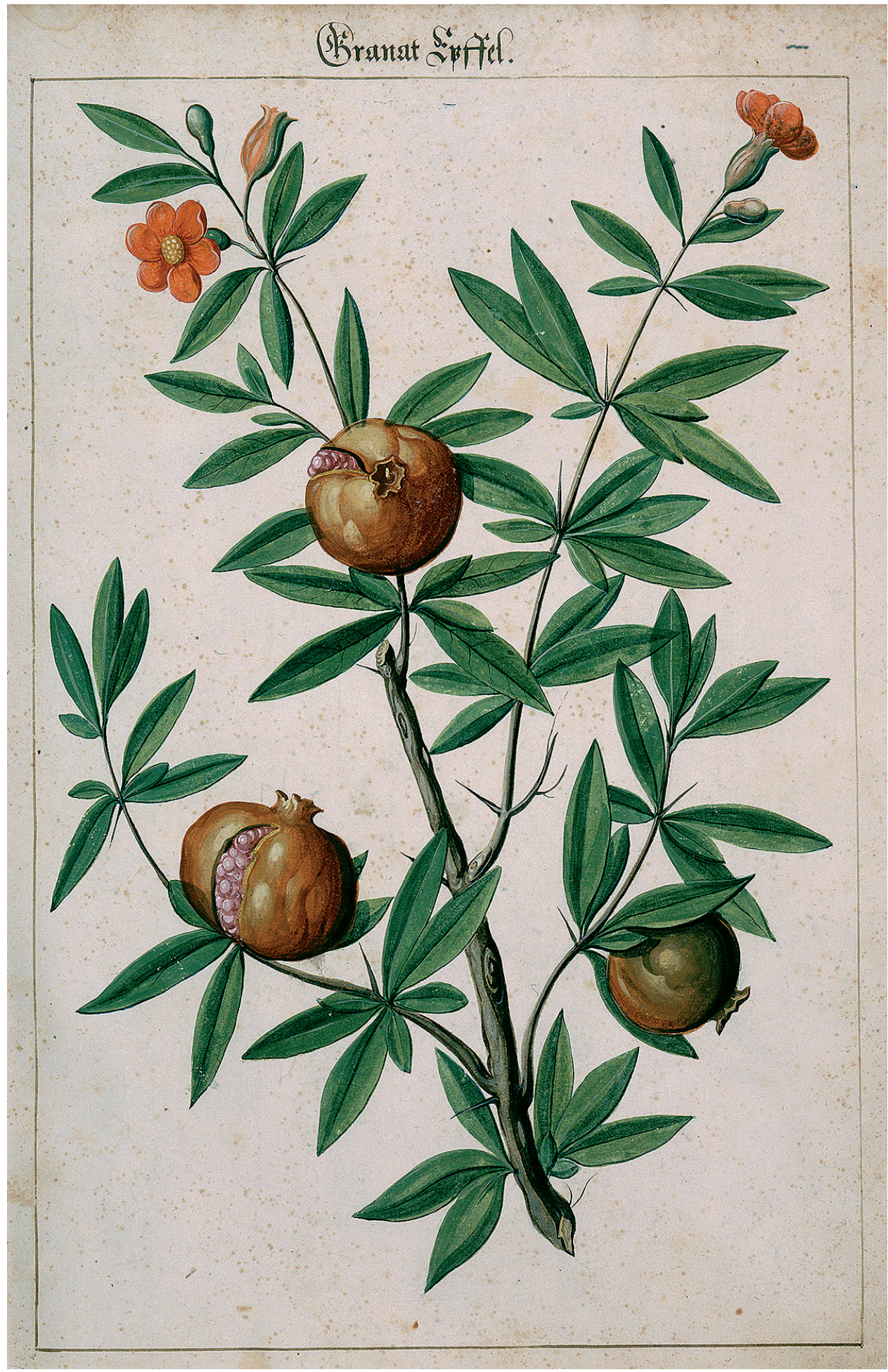

1 | Granatapfelbaum, Wasserfarben, circa 483 x 340 mm; aus Kentmann, Johannes: Kreutterbuch, Torgau 1563 (Staats- und Universitätsbibliothek Dresden, Handschriftensammlung, Mscr.Dresd.B71, Bl. 7r). 
des Hofes eingerichtet worden war, zuständig. Seine Aufgabe war unter anderem die Erzeugung und Pflege der für den täglichen Verbrauch in der Küche notwendigen Gemüse, Früchte und Kräuter."

Die Kurfürstin nutzte die Gärten in Torgau, Lochau und Dresden zur Versorgung der Hofküche. Für die Dresdner Gärten, die sich direkt am Schloss, im »breiten Zwinger« sowie im Vorwerk Ostra befanden, erhielt das Kurfürstenpaar unter anderem »Stauden von großen Johannisberlein« sowie Rübensamen von der bayerischen Herzogin Anna, Erzherzogin Maria von Österreich sandte Salat und Krautsamen sowie spanische und türkische Gewächse, Landgräfin Sabina von Hessen-Kassel ließ Melonensamen und andere »seltsame« Pflanzen aus Italien überbringen, Erzbischof Johann Jacob von Salzburg schickte Samen und Zweige von italienischen Früchten sowie Salatpflanzen, nämlich Endivien, Chicoree und Portulak. ${ }^{12}$

Kurfürst August übte sich seit spätestens 1571 als äußerst praktisch veranlagter Souverän intensiv im Säen, Setzen und Pfropfen - also Veredeln - von Obstbäumen. Der Grund lag unter anderem darin, dass der Obstbau in Sachsen wenig entwickelt war und die Versorgung der im Bergbau tätigen ländlichen Bevölkerung sowie derjenigen in den größeren Städten durch die Einfuhr von Obst und Gemüse gedeckt werden musste. Der Kurfürst, stets auf die Stärkung und Entwicklung der eigenen Wirtschaft bedacht, widmete sich daher intensiv der Förderung einheimischer Produkte und der Verbesserung der Anbaumethoden.

Im Sommer 1571 hielt sich Kurfürst August $\mathrm{zu}$ Besuch bei Markgraf Georg Friedrich in Ansbach auf. Dabei lernte er nicht nur den in Franken gut entwickelten Obstanbau kennen, sondern bewunderte auch die markgräflichen Obstgärten. Er erbat sich vom Herzog 2.000 junge Obstbäume. Die Fuhre mit eintausend Apfel- und eintausend Birnbäumen erreichte Sachsen im Oktober 1571. ${ }^{13}$

Bereits im Juli 1571 hatte Kurfürst August einen Baumgärtner namens Adam Wolf aus Nürnberg nach Dresden geholt,

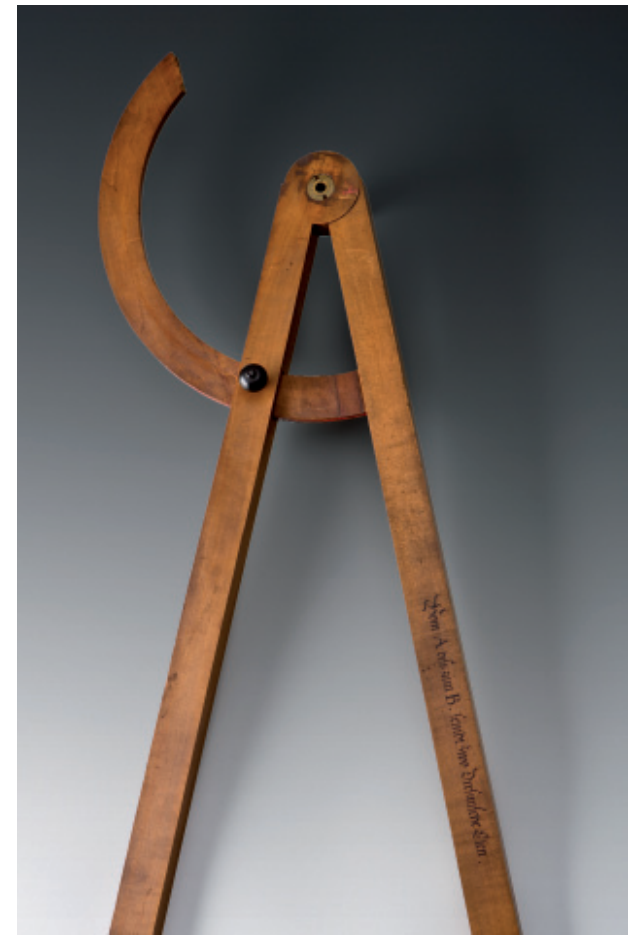

2 | Freilandzirkel, Deutschland um 1560-1570, Birnbaum, Ebenholz, Messing, Eisen, rotes Wachs, Länge $128 \mathrm{~cm}$ (SKD, Rüstkammer, Inv.-Nr. P 102).

der für die Aufzucht von Obstbäumen und die Anlage von Obstbaumgärten zu sorgen hatte. ${ }^{14}$ Der Baumgärtner wohnte im Vorwerk Ostra in Dresden und legte dort eine Baumschule an. Seine Aufgaben sind in der Bestallungsurkunde genau spezifiziert: Neben dem "Zurichten « und Düngen des Bodens, sollte er eine große Anzahl Samen von verschiedenen Obstsorten sowie etliche tausend wilde Obststämme zusammentragen, um diese im Herbst zu säen bzw. in der Baumschule einzupflanzen. Zur rechten Zeit sollten diese wilden Stämme dann mit dem besten Obst, das heißt Reisern davon, gepfropft werden. ${ }^{15}$ Der Bestallungsurkunde für den Baumgärtner kann man entnehmen, dass sich zu diesem Zeitpunkt reine Obstgärten in Torgau, Lochau (dem späteren Annaburg) und in Stolpen befanden. Die Baumschule zur Anzucht der Obstbäume lag hingegen im Vorwerk Ostra in Dresden. Der neue Baumgärtner Adam Wolf be- 


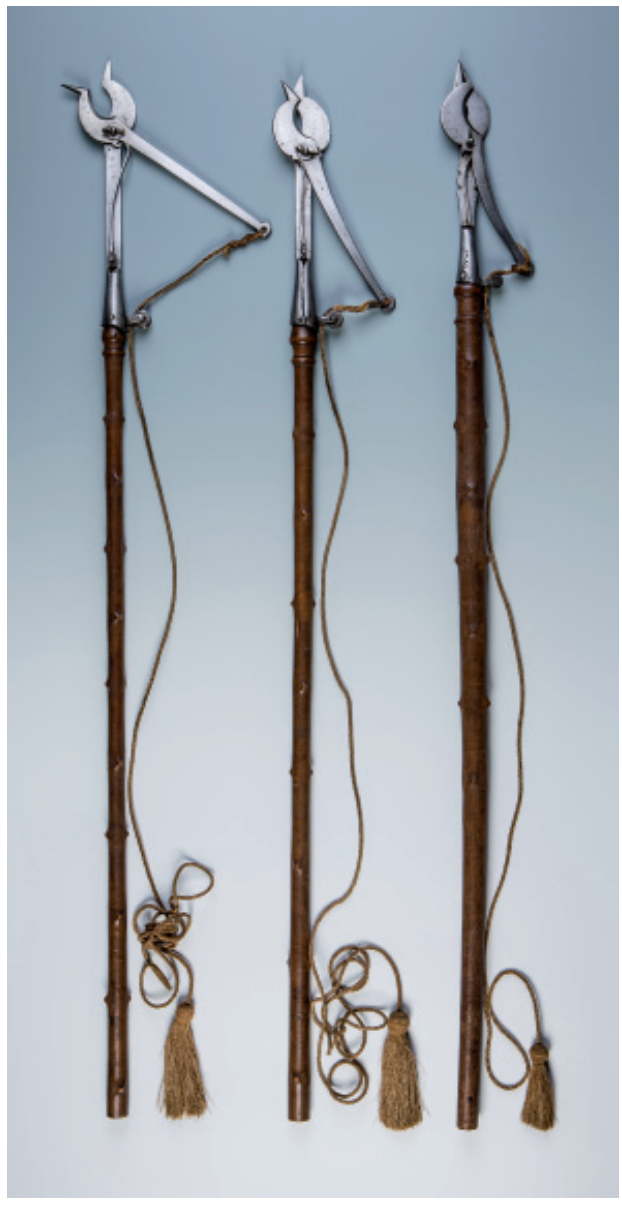

3 | Baumscheren, Deutschland, um 1560-1570, Eisen, Holz (Birnbaum?), Hanf, Länge zw. 112,8 und $116 \mathrm{~cm}$ (SKD, Rüstkammer, Inv.-Nr. P 93, P 92, P 91).

richtete dem Kurfürsten August bei seiner Ankunft, dass er "auf seinem garten in Nürnbergk biß in 4000 gepfropfte junge baum von allerley des besten obst hette, welche er sonst verkauffen « müsste. Diese bot er dem Kurfürsten zum Erwerb an. Die Bäumchen wurden im Herbst 1572 nach Dresden gebracht. ${ }^{16}$

Zum ersten Mal erging im Jahr 1571 der Befehl an verschiedene Amtsverwalter und Forstmeister, wilde Obstkerne und junge, wilde Obstbaumstämme zu sammeln. Die Kerne sollten sortiert abgeliefert werden. Als Gegenleistung erhielten die Lieferanten pro Scheffel Kerne einen Scheffel Roggen. ${ }^{17}$ Die zusammengetragenen Kerne, so berich- tete Bartel Lauterbach am 1. November 1571, habe der Gärtner Georg Winger »bei der Willischen Bastei aufgeschüttet und ausgebreitet«, so dass sie nicht zu dick übereinander liegen. Täglich »rühre« er sie um, damit sie nicht verderben. ${ }^{18}$ Im August 1572 konnte Georg Winger dem Kurfürsten vermelden, dass in Ostra von den 32 Scheffeln ausgesäten Apfel- und Birnenkernen die meisten etwa eine halbe Elle hoch gewachsen seien. ${ }^{19}$

Kurfürst August beschäftigte sich selbst mit dem Veredeln von Obstbäumen. Seine Erfahrungen brachte er in einem »Künstlich Obst- und Gartenbüchlein« zu Papier und gab es 1571 zum Druck frei. Diese erste Auflage ist nicht mehr nachweisbar, doch wenigstens drei Nachauflagen aus dem 17. Jahrhundert sind bekannt. ${ }^{20}$ Der Kurfürst beschreibt hierin zunächst die Unterschiede der verschiedenen Baum- und Fruchtsorten, lässt sich ausführlich über die diversen Arten des Pfropfens und Veredelns aus und berichtet über die Pflege der Bäume, darunter das Ausästen und das Entfernen von Raupennestern und anderen Schädlingen. ${ }^{21}$ Die hierzu notwendigen Gerätschaften ließ sich der allseits interessierte Fürst auch gleich liefern. Vermutlich entstanden die später in der Kunstkammer aufbewahrten Gartengeräte in Nürnberg, wo auch die Gärtner herkamen. Um gleichmäßige Abstände beim Pflanzen von Samen, Absenkern oder Bäumchen zu erhalten, waren Freilandzirkel dienlich, die sich ebenso für das Abstecken und Einteilen von Pflanzflächen, Beeten und Rabatten eigneten. Der fixierbare Stellbogen garantierte gleich bleibende Distanzen. Ein aus Birnbaumholz gearbeitetes Stück konnte bis zu zwei Dresdner Ellen messen (Abb. 2). Zur Pflege der Obstbäume setzte man sogenannte Raupenscheren ein, die mittels eines Hanfstrickes auch weit über dem Kopf wachsende Zweige erreichten (Abb. 3).

Für das Jahr 1572 überliefert eine Art Tagebuch, dass sich der Kurfürst an Pfropfungen und Aussaaten versuchte. Hierin sind auch einige Zugänge an Obstbäumchen 


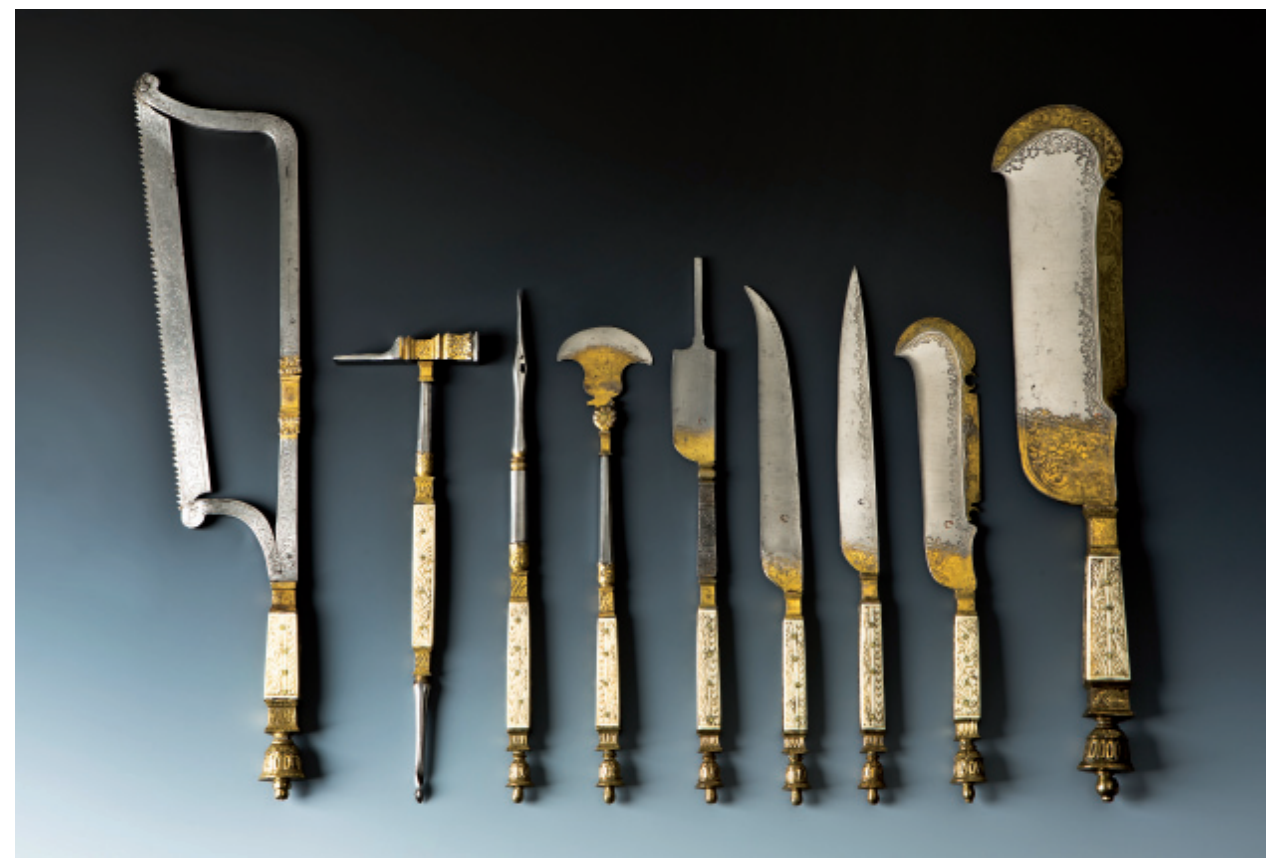

4 | Gartenbesteck oder Pfropfzeug mit Griffen aus Eichenholz und Elfenbein, Frankreich oder Deutschland in französischem Stil, um 1575, Geschenk der Markgräfin Katharina von Brandenburg, Eisen, teilweise vergoldet, Eichenholz, Elfenbein, L. max. 35 cm (SKD, Rüstkammer, Inv.-Nr. X 555).

und Reisern verzeichnet. Der Pfalzgraf Johann Casimir sandte beispielsweise eine unbekannte Anzahl von Reisern in 102 Sorten und der Oberst Heinrich von Gleisenthal schickte 307 Reiser in 21 Sorten..$^{22}$ Alle Reiser wurden im Frühjahr 1572 auf vorbereitete junge wilde Obststämme gesetzt. ${ }^{23}$ Der Kurfürst selbst pfropfte 125 Apfelreiser, die der Oberst Heinrich von Gleisenthal gesandt hatte, »im gartten nicht weit vom grünen gewelbe", also an der Westseite des Dresdner Schlosses. ${ }^{24}$

Dass der Kurfürst Hand anlegte, ist auch der Korrespondenz zu entnehmen, so beispielsweise dem Dankesschreiben der Kurfürstin Anna an den Salzburger Erzbischof von 1574. Zu den übersandten Pfropfreisern vermerkte die Kurfürstin:

»es hat auch unser hertzliebster $\mathrm{h}$ [err] und gemahel solche pelzreißer mehrern theils selbst aufgesezt und gepropfet, und sein der zuvorsicht, e[uer] l[ieben] werde dermal eins unsern herzliebsten herrn und gemahel selbst persönlich alhier be- suchen, domit sie sehen, wie solche reiser [...] kommen unde gewachsen, und der früchte davon selbst genießen mögen. «25

Experimentierfreudig ging der Kurfürst an die Sache heran. In einem Dokument von 1572 ist genau aufgeführt, welche Obstsorten der Kurfürst auf welche Stämmchen setzte: Kastanienzweige wurden auf Maulbeerbaum oder Nussbaum gepfropft, Mispeln wurden auf Birnbaum, Mandeln auf Maulbeerbaum und Haselnuss auf Walnussbäumchen gesetzt. ${ }^{26}$

Zum Pfropfen sind Sägen und diverse Messer nötig (Abb. 4). In der Werkzeugsammlung des Kurfürsten, die spätestens seit 1572 als Kunstkammer bezeichnet wurde, haben sich einige fürstliche Gartenbestecke mit zahlreichen Messern erhalten. Sie waren meist Geschenke an den gärtnernden Kurfürsten und sind zum Teil viel zu aufwendig verziert, als dass sie zum tatsächlichen Gebrauch gedacht waren. Doch sie verweisen auf dessen Leidenschaft. Aus 
dem ersten Kunstkammerinventar von 1587 ist zudem bekannt, dass der Kurfürst einen zusammenfaltbaren Pfropfstuhl besaß. ${ }^{27}$ Er kam wohl bei Veredlungen an niedrigen Wildstämmchen zum Einsatz.

Das Tagebuch von 1572 gibt weiterhin Auskunft darüber, was der Kurfürst säte oder steckte, z.B. Granatapfelkerne, Buschbohnen (»welsche bohnen«), Kastanien, Johannisbrotkerne (Johannisbrotbaum), Kirschkerne, Aprikosenkerne (Morellen), Zirbelkiefer-, Sonnenblumen- und Tamarindenkerne. ${ }^{28}$ Für diese Aussaaten im Garten ließ sich der Kurfürst zwei nützliche Geräte anfertigen, die ihm die Arbeit erleichterten (Abb. 5). Die beiden Kernsetzer aus der Kunstkammer dürften direkt nach Angaben des Herrschers hergestellt worden sein und erlaubten das Säen von Obstkernen ohne lästiges Bücken, selbst vom Pferd aus. Die mit 1572 datierten Messingstäbe sind sowohl mit den Initialen des Kurfürstenpaares "AA " (für August und Anna) als auch mit dem Anfang des Lutherchorales "Erhalt uns

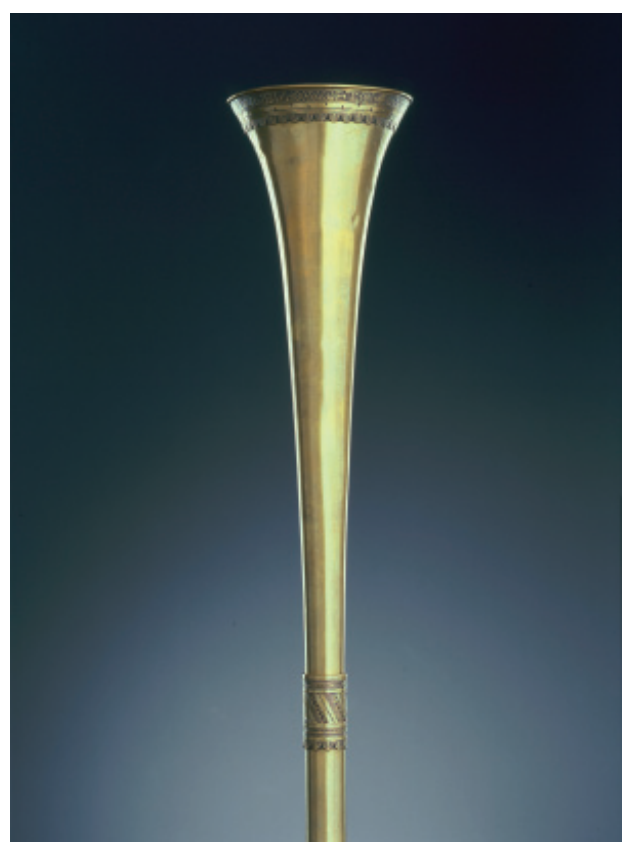

5 | Kernsetzer, zu Fuß zu gebrauchen, wohl Dresden, datiert 1572, Messing vergoldet, Länge 110,6 cm (SKD, Rüstkammer, Inv.-Nr. P 95).
Herr bei Deinem Wort« verziert. Der Kurfürst soll die beiden Geräte tatsächlich selbst benutzt haben. ${ }^{29}$

$\mathrm{Zu}$ Tausenden gelangten in den kommenden Jahren junge bereits gepfropfte Obstbäume, Obstbaumreiser und wilde Stämme zum Veredeln sowohl als Geschenke vom Kaiser und befreundeten Fürsten sowie als Ankäufe nach Sachsen. ${ }^{30}$ Der kursächsische Rat Hans Christoph von Bernstein (15221580) bot dem Kurfürsten im August 1573 wohl aus eigenen Gärten 200 bereits gepfropfte Obstbäume an. Diese dürften besser an das Klima angepasst gewesen sein als die begehrten italienischen oder süddeutschen Gewächse. Unter den Apfelsorten befanden sich u.a. Borsdorfer, Hartig, Zapfenapfel, Musapfel und der Weißling. Unter den Birnensorten waren Speckbirnen, Michelsbirnen, Wasserbirnen, Paris- und Honigbirnen zu finden. ${ }^{31}$

Die Pfropfungen wurden in den Dresdner Gärten und vor allem in der Baumschule im Ostra-Vorwerk vorgenommen, die bereits veredelten Bäumchen je nach Wuchs zur Aufzucht in der Baumschule oder direkt in die Obstgärten in Dresden und Stolpen gesetzt. 1577 konnte Georg Winger dem Kurfürsten vermelden, dass sich mittlerweile 16.00o Bäume im Zwingergarten und in der Baumschule in Ostra befanden..$^{32}$

Ungefähr um 1576 begann der Kurfürst mit der Planung einer riesigen Obstbaumanlage in Annaburg. Das ehemalige ernestinische Jagdschloss Lochau war 1572-1575 durch den Neubau der Annaburg ersetzt worden. Direkt am Schloss lagen der Gemüse- und der Arzneipflanzengarten. Der "Neue Garten« mit Obstbäumen nahe am Schloss war der bereits von Friedrich dem Weisen angelegte Lustgarten. Kurfürst August ließ im sogenannten Tierpark, der an all diese Gartenanlagen anschloss, eine kreisförmige Grundfläche im Durchmesser von etwa 1600 Ellen roden (Abb. 6). Die Arbeiten erfolgten mit Hilfe von Hunderten Arbeitskräften. Im Dezember des Jahres 1577 befahl Kurfürst August dem Baumgärt- 
ner Georg Heinrich, dass er für die bis dahin gesammelten 26 Scheffel Haselnüsse, 15 Scheffel Kirsch- und 14 Scheffel Apfelkerne einen Platz im großen Tiergarten bestimmen möge, wo die wilden Bäume bereits gerodet waren. ${ }^{33}$ Mit Hilfe von Arbeitern und Pferden sollte dort der Boden durch Umgraben und Düngen so vorbereitet werden, dass man die Kerne im nächsten Frühling aussäen konnte. Im Herbst 1578 kamen außerdem 30.000 Bäumchen in Annaburg an und wurden vom Hofgärtner Georg Winger mit fünf Gehilfen gepflanzt. ${ }^{34}$ In den 1590er Jahren sollen sich 266.850 Bäume in dem Obstgarten zu Annaburg befunden haben, inklusive der zur Begrenzung der Wege und der Rundung gesetzten Bäumchen. ${ }^{35}$

Einige Gerätschaften, die zur Urbarmachung von noch ungerodeten Flächen dienlich sind, bewahrte der Kurfürst in seiner Kunstkammer auf. Hierzu gehören diverse Hacken, vor allem stabile Rodehacken, Spitz- und Breithacken, aber auch Spaten (Abb. 7). Ein Teil der in der Kunst- kammer aufbewahrten Gartengerätschaften hat als Besonderheit aus Obstbaumholz gedrechselte Stiele, unterscheidet sich ansonsten aber wenig von heutigen Geräten. Die erhaltenen etwa 50 Gartenwerkzeuge, werden in der Dauerausstellung »Weltsicht und Wissen um 1600" im Dresdner Georgenbau präsentiert.

Während sich Kurfürst August mit dem Veredeln von Obstbäumen und der Anlage großflächiger Obstanlagen beschäftigte, gehörte - im Zusammenhang mit der Gärtnerei - die Verarbeitung und Haltbarmachung von Obst neben der Herstellung von Arzneien und dem berühmten Aquavit zu den Hauptaufgaben der Kurfürstin Anna. Seit dem Neubau von Schloss Annaburg entwickelte sich dieses Schloss zur Lieblingsresidenz des Kurfürstenpaares. Dort befanden sich eine Drechselstube und das Laboratorium des Kurfürsten sowie die Bibliothek des Paares, in der auch gedruckte und handgeschriebene Gartenbücher aufbewahrt wurden. $\mathrm{Zu}-$

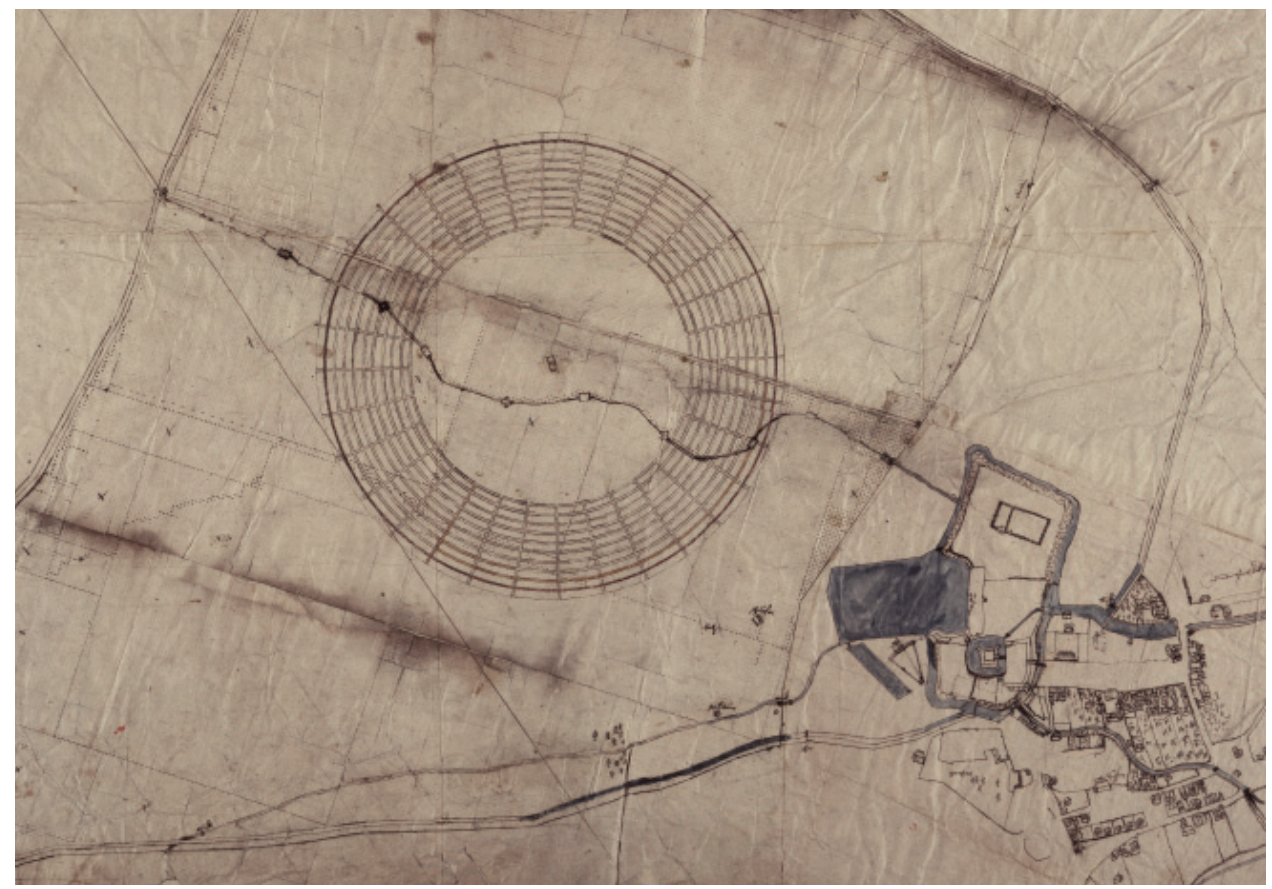

6 | Ausschnitt aus: Abriss des Baumgartens zu Annaburg, 1578 (SächsHStAD, 12884 Karten und Risse, Schrank 5, Fach 68, Nr. 9dd). 
dem errichtete die Kurfürstin hier eine neue und leistungsfähige Anlage zum Destillieren von Kräutern und Gewürzen, um medizinische Tinkturen und Essenzen herzustellen. ${ }^{36}$ Auch ihr Aqua Vitae, das einen zweijährigen Destillierprozess durchlief und mit zahlreichen Zusätzen versehen wurde, entstand in dem Destillierhaus. Daneben wurde jedes Jahr Obst in großen Mengen unter Aufsicht und Anleitung der Kurfürstin haltbar gemacht und gelagert. Aus der umfangreichen Korrespondenz der Kurfürstin geht hervor, dass sie an einheimischen Obstsorten vor allem Quitten, Birnen, Äpfel, Pflaumen, Pfirsiche und Kirschen verarbeitete. Zusätzlich eingeführt wurden Zitronen, Tamarinden, Pomeranzen (Bitterorangen), Granatäpfel, Datteln und neue Quittensorten. ${ }^{37}$ Aus den einheimischen Früchten bereitete die Kurfürstin Saft und Latwerge (ein mit Honig oder Zucker angesetztes und durch langes Kochen eingedicktes Fruchtmus, das auch als Soßengrundlage verwendet werden konnte) sowie Konfekt (ein noch weiter eingekochtes und am Ende getrocknetes Fruchtmus, das sich schneiden ließ). Daneben wurden auch Äpfel, Birnen und Pflaumen gedörrt. ${ }^{38}$ Vor allem die Verarbeitung von Quitten scheint der Kurfürstin besonders gut gelungen zu sein, wie Landgräfin Sabina von Hessen einmal bestätigte, als Anna ihr Quittensaft zukommen ließ, »er ist in Wahrheit schön, man muss e[uer] 1[ieben] meister sein lassen, e[uer] 1 [ieben] habens besser gelernt, als ichs selber kann. « ${ }^{39}$

Doch die in Sachsen wachsenden Quittenbäume brachten nicht genügend Ausbeute. So schrieb der Hausmarschall Hans von Auerswalde 1575 an die Kurfürstin, dass die Quitten im Dresdner Zwinger zwar geblüht, aber keine Früchte angesetzt hätten. ${ }^{40}$ Die Kurfürstin bat daraufhin unter anderem Brigitta Trautson, die Gemahlin des Oberhofmarschalls am Kaiserhof in Wien, um Quitten, welche diejenigen »hier zu land an geruch, geschmack, güte und größe bei weitem übertreffen " und erhielt diese auch. ${ }^{41}$ Quitten- und Mispelreiser übersendete 1568 die Markgräfin Katharina von Branden-

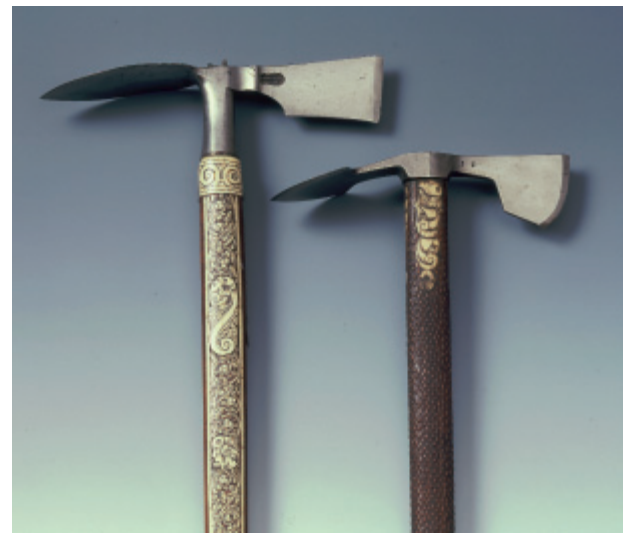

7 | Rodehacken, Deutschland, um 1560-1570, Eisen, Holz, Bein, Länge 90,6 und 95,5 cm (SKD, Rüstkammer, Inv.-Nr. P 76, P 75).

burg-Küstrin. ${ }^{42}$ Große Quitten kamen auch von Erzherzog Ferdinand von Tirol. ${ }^{43}$ Den Schösser von Senftenberg wies Anna 1580 an, darauf zu achten, dass ihre Untertanen die geernteten Quitten nicht etwa nach Leipzig oder andernorts verkauften, sondern sie der Kurfürstin für sechs bis sieben Groschen das Schock (6o Stück) überließen. ${ }^{44}$

Über die Tätigkeit der Kurfürstin erfährt man vor allem aus ihrer Korrespondenz. Vielfach wurde sie um Produkte und Rezepte gebeten, häufig verschenkte sie diese und erhielt entsprechende Dankschreiben. So erbat sich Katharina von Brandenburg 1559 von Kurfürstin Anna das Rezept für Quittenmus, für klaren Quittensaft und Johannisbeersaft. ${ }^{45}$ Die Herzogin von Mecklenburg erhielt auf ihr Ansuchen 1575 das Rezept "kirschen einzumachen « und die Gräfin von Henneberg wünschte im gleichen Jahr das Rezept, wie man die Johannisträublein einmacht. ${ }^{46}$ Die Bandbreite an Verarbeitungsarten und die eingemachten Obstsorten zeigt eine Geschenksendung, die Anna an ihre Mutter Dorothea, die dänische Königin, im Jahr 1556 übersandte. ${ }^{47}$ Sie bestand aus einem großen zinnernen Ständer mit eingemachten Quitten, einem solchen Ständer mit eingemachten Pfirsichen, je einem mittelmäßig großen Ständer mit Kirschmus, mit Weinbeermus, mit Kirschsaft, mit Pfirsichsaft und einem mit Quitten- 
saft sowie vier Waldenburger Krügen mit Quittenlatwerge. 20 Jahre später, im Jahr 1575, gab die Kurfürstin ihrer dänischen Hofdame Anna von Hardenberg, die eigentlich zum Hofstaat ihrer 1571 verstorbenen Mutter gehört hatte, eine Reihe von Produkten nach Dänemark mit: darunter in »ein $[\mathrm{em}]$ ladlein ezliche schachteln [...] mit quittensaft, quitten lattwerge, quitten zeltten, eingemachte pommeranzen schalen, heiße magen latwerge und dergleichen eingemachtes mehr $[\ldots] \ll$. $^{48}$

Dieser nur sehr beschränkte Einblick in die Tätigkeiten der Kurfürstin zeigt, dass sich das Kurfürstenpaar, wie in so vielen anderen Bereichen, auch in ihrem Interesse für Garten- und Obstbau hervorragend ergänzte.

\section{Resümee}

$\mathrm{Zu}$ den zahlreichen Interessen des Kurfürsten August zählte die Beschäftigung mit der Landwirtschaft und dem Gartenbau. Ein besonderes Faible entwickelte er jedoch für den Obstanbau, dem er sich spätestens seit 1571 intensiv widmete. Wie auch in anderen Gewerken legte der Fürst selbst Hand an, um die Technik des Pfropfens zu erlernen und sich im Säen und der Aufzucht von Obstbaumsorten zu üben. Er suchte sich zudem

1 Hoppe, Stephan: Anatomy of an Early „Villa“ in Central Europe. The Schloss and garden of the Saxon Elector Frederick the Wise in Lochau (Annaburg) according to the 1519 Report of Hans Herzheimer, in: Monique Chatenet (Hg.): Maisons des champs dans l'Europe de la Renaissance, Paris 2006, S. 159-170, besonders S. 160-164.

2 Bürger, Thomas: Das Kräuterbuch des Johannes Kentmann von 1563, München/Berlin/London/New York 2004, S. 8.

3 Der Begriff Lustgarten taucht später ebenfalls in Bestallungsurkunden und der Korrespondenz von Kurfürst August und Kurfürstin Anna auf. Es handelt sich dabei offenbar um Anlagen, die sowohl Obstbäume, Sträucher, Kräuter und Blumen enthielten und damit eine Mischung aus Nutzgarten und Ziergarten waren. Neben der Verwertung der dort angebauten Gemüse, Früchte und Kräuter in der Hofküche, dienten die Gärten auch der Erholung und waren Orte, an denen das Kurfürstenpaar persönlichen Interessen, wie dem Pfropfen oder fähige und vertrauenswürdige Gärtner, in deren Hände er die Weiterentwicklung und Förderung von Maßnahmen zur Ausweitung des Obstanbaus und zur Ertragssteigerung legte.

All diese Bemühungen begrenzten sich auf den Rahmen der fürstlichen Gärten, auch wenn diese in Stolpen und Annaburg eine enorme Größe erreichten. Eine Anweisung des Kurfürsten weist jedoch auf dessen eigentliches Ziel hin: den Obstbau in ganz Sachsen entscheidend voranzubringen. Die Anweisung besagte, dass jedes Paar, das zu heiraten gedachte, zwei Obstbäume pflanzen musste. Dieser Befehl ist zwar nicht mehr im Original nachweisbar, aber ein Mandat $\mathrm{Au}-$ gusts des Starken aus dem Jahr 1700 bezieht sich auf die Verordnung des 16. Jahrhunderts. Darin stellte August der Starke fest, dass er sich der Anweisung des Kurfürsten August zur Pflanzung guter Obstbäume und anderer nutzbarer Stämme anzuschließen gedenke und befahl, dass jeder Bräutigam (egal, ob junger Geselle oder Witwer) vor der Heirat sechs Obst- oder andere Nutzbäume gepflanzt oder gepfropft haben musste. Nur bei Vorweisung einer entsprechenden Bescheinigung vom Amtmann oder einem Gerichtsherrn durften die Pfarrer die Trauung vornehmen.

der Aufzucht exotischer Gewächse (Tabak, Mais, Tulpen) nachging.

4 Nagel, Christine: »Suche [...] mitt großem verlangen undt lust auslendsche samen. «Die Gärtnerei unter dem Kurfürstenpaar August und Anna von Sachsen, in: Staatliche Kunstsammlungen Dresden (Hg.): Flora, Kunstblatt, 61. Jg., Dresden 2018, S. 17

5 Ebd., S. 17-18.

6 Ebd., S. 19-21.

7 Kentmann, Johannes: Kreutterbuch, Torgau 1563, Bürger 2004.

$8 \mathrm{Zu}$ den botanischen Gärten in Meißen und Torgau, siehe Lange, Karl-Heinz: Zur Geschichte des Medizinwesens, Torgau 1997, S. 17 und S. 19-21.

9 SächsHStAD, 10004, Nr. 513, Kopial der Kurfürstin Anna, fol. 108r.

10 SächsHStAD, 10004, Nr. 513, fol. 131r.

11 Bestallungsurkunde (Konzept) als Hofgärtner für Georg Winger, 20.12.1568, SächsHStAD, 10004 (Kopiale), Nr. 343, Kopial des Kurfürsten August $1568 / 69$, fol. 444 r- 445 r. 
12 Weber, Karl von: Anna Churfürstin zu Sachsen, geboren aus königlichem Stamm zu Dänemark. Ein Lebens- und Sittenbild aus dem 16. Jahrhundert, Leipzig 1865, S. 131; Nagel 2018, S. 19.

13 Markgraf Georg Friedrich aus Onolzbach (Ansbach) an Kurfürst August, 3.9.1571, SächsHStAD, 10024 (Geheimes Archiv), Loc. 8601/20, Allerlei Schreiben und Berichte über die Hofgärtnerei 1571-1573, fol. 3r-v.

14 Bestallungsurkunde (Konzept) als Baumgärtner für Adam Wolf, 17.7.1571, SächsHStAD, 10004, Nr. 223, Kopial des Kurfürsten August, 7r-8r.

15 Ebd.

16 SächsHStAD, 10004, Nr. 467, Kopial des Kurfürsten August, fol. 277r.

17 Falke, Johannes: Die Geschichte des Kurfürsten August von Sachsen in volkswirtschaftlicher Beziehung, Leipzig 1868, S. 115. Eine ähnliche Anweisung erging auch 1574 an den Schösser von Dresden: SächsHStAD, 10047 (Amt Dresden), Nr. 4418, Das Sammeln und Abliefern von Kirschkernen betr. 1574, unfol.

18 SächsHStAD, 10024, Loc. 8601/20, fol. 1or.

19 Ebd., fol. 64r.

20 Künstlich ObstGarten Büchlein, Churfürst Augusti zu Sachsen. Auffs New von einem Liebhaber des Garten Bawes wiederumb an tag geben, Berlin 1619. 21 Ebd.

22 Vortzeichnus zu welcher zeitt der churfürst zu saxen [...] wohl Dresden ca. 1571, SLUB, Mscr.Dresd.K340, fol. 26r und 27v (Apfel- und Birnenreiser von Oberst Heinrich von Gleißenthal), fol. 26v, 28r und $31 \mathrm{v}$ (Äpfel, Birnen und Kirschen von Pfalzgraf Johann Casimir).

23 Vortzeichnus [...] 1571, Bl. 1r-25V.

24 Ebd., fol. 26r. Dieser Garten an der Westseite des Schlosses entstand bereits 1545, erfuhr jedoch ab 1569 durch eine Vergrößerung der Festung im Nordwesten des Schlosses eine Erweiterung, die offenbar eine Anpflanzung von Obstbäumen in größerer Zahl ermöglichte. Der Garten schloss unmittelbar an die Räume im Erdgeschoss an, die heute das Weißsilberzimmer und den Pretiosensaal des Historischen Grünen Gewölbes bilden. Siehe: Butenschön, Sylvia: Geschichte des Dresdner Stadtgrüns, in: Forum Stadt- und Regionalplanung e.V. (Hg.): Arbeitshefte des Instituts für Stadt- und Regionalplanung, Heft 68, Berlin 2007, S. 27.

25 Kurfürstin Anna an den Erzbischof von Salzburg, 13.4.1574, SächsHStAD, 10004, Kopial der Kurfürstin Anna, fol. 168v.

26 Varia Oeconomica, 16. Jahrhundert, SLUB, Mscr. Dresd.K.68, fol. 26r, Verzeichnis auf wie vielerley art der Kurfürst zu Saxen, Herzog Augustus, den 13. marty 72 zu Dresden gepfropfet.

27 SächsHStAD, 10009 (Kunstkammer, Sammlungen, Galerien), Nr. 1, Inventar Kunstkammer 1587, fol. 291r/343r.

28 Vortzeichnus [...] 1571, Bl. 1r-25v.

29 SächsHStAD, 10009, Nr. 1, Inventar Kunstkammer 1587 , fol. $205 \mathrm{v} / 215 \mathrm{v}$.
30 Dutschmann, Georg: Aus Georg Wingers Tätigkeit als Hofgärtner des Kurfürsten August von Sachsen, in: Sitzungsberichte und Abhandlungen der Flora, Neue Folge, 36-38, 1931-1933 [1934], S. 80-86, hier S. 8o. Georg Dutschmann listet hintereinander die im Jahr 1573 eingegangenen Pfropfreiser des Kaisers Maximilian (vier Lieferungen), der Herzogin von Liegnitz und Brieg, des Pfalzgrafen Johann Casimir sowie eine Lieferung aus Breslau, Krakau und Jägerndorf auf. Die ausführlichen Listen mit den Namen der Obstsorten finden sich in: SächsHStAD, 10024, Loc. 8601/20, fol. 34r-46r.

31 SächsHStAD, 10024, Loc. 8601/20, fol. 48-49r. Ein Teil der Sortennamen ist zu finden in: Gürtler, H.: Apfelnamen aus dem 16. Jahrhundert, Zeitschrift für deutsche Wortforschung 12 (1910), S. 215-222 und ders.: Birnennamen des 16. Jahrhunderts, ebd., S. 248-254.

32 SächsHStAD, 10024, Loc. 8601/20, fol. $76 \mathrm{v}$.

33 Kurfürst August an den Schösser von Annaburg, 13.12.1577, SächsHStAD, 10004, Nr. 432, Kopial des Kurfürsten August 1577, fol. 381r. Der Scheffel ist ein altes Raummaß, das in Dresden vor 1840 bei etwas über 100 Litern für einen Scheffel lag. Siehe Verdenhalven, Fritz: Alte Meß- und Währungssysteme aus dem deutschen Sprachgebiet, Neustadt/Aisch 1993, S. 50.

34 Gründler, Ernst: Schloß Annaburg. Festschrift zur einhundertfünfzigjährigen Jubelfeier des MilitärKnaben-Instituts zu Annaburg, Berlin 1888, S. 81. 35 Ebd.

36 Peickert, Heinz: Gräfin Dorothea von Mansfeld und Kurfürstin Anna von Sachsen. Zwei deutsche Arzneimittelfabrikantinnen des 16. Jahrhunderts, in: Apotheker-Zeitung, Nr. 48 (1933), S. 87-89.

37 Röhner, Regine: Eine Kurfürstin in der Küche. Anna von Sachsen und ihre Rezepte, Leipzig 2012, S. 51.

38 Weber 1865 , S. 115.

39 Ebd., S. 114-115.

40 Ebd., S. 137.

41 Kurfürstin Anna an Birgitta Trautson, 20.10.1563, SächsHStAD, 10004, Nr. 511, Kopial der Kurfürstin Anna, fol. 115v.

42 SächsHStAD, 10024, Loc. 8531/03, Frau Katharinas, Markgraf Johanns [(Hans) von Küstrin] zu Brandenburg Gemahlin, Briefe an die Kurfürstin [Anna] zu Sachsen, 1567-1574, fol. 1r. Zu den Mispeln auch Weber 1865, S. 137.

43 Kurfürst August an Erzherzog Ferdinand von Tirol, 23.4.1575, SächsHStAD, 10004, Nr. 404, Kopial des Kurfürsten August, fol. 96v.

44 Weber 1865, S. 158; Röhner 2012, S. 51.

45 Katharina von Brandenburg, geb. von Braunschweig, an Kurfürstin Anna, 24.9. 1559. SächsHStAD, 10024, Loc. 8528/o1, Schreiben an die Kurfürstin Anna, [1554-1561], fol. 202r.

46 Weber 1865, S. 101.

47 Ebd., S. 115.

48 Kurfürstin Anna an Anna von Hardenberg, 7.11.1575, SächsHStAD, 10004, Nr. 518, Kopial der Kurfürstin Anna, fol. 123r. 